\title{
Espectros de gotas e idade de trifólios e sua interação com a área abaixo da curva de progresso da ferrugem da soja
}

\author{
Drops spectra and leaflets age and its interaction with the area under disease progress curve of soybean rust
}

\author{
Giuvan Lenz $^{I^{*}}$ Ricardo Silveiro Balardin $^{\mathrm{I}}$ Simone Gripa Minuzzi ${ }^{\mathrm{I}}$ Marcelo Madalosso $^{\mathrm{I}}$ Monica \\ Debortoli $^{\mathrm{I}}$ Caroline Gulart ${ }^{\mathrm{I}}$ Juliano Perlin de Ramos ${ }^{\mathrm{I}}$
}

\section{RESUMO}

O objetivo do presente trabalho foi avaliar o efeito de espectros de gotas (grossas, médias e finas) na velocidade de absorção de fungicidas, para trifólios de diferentes idades, através de medida indireta expressa pelo residual de controle de ferrugem asiática da soja (Phakopsora pachyrhizi). O delineamento experimental utilizado foi inteiramente casualizado, com quatro repetições, em arranjo fatorial (3x3x5x4), cujos fatores foram compostos por: três espectros de gotas (grossas, médias e finas); três idades fisiológicas de trifólios na planta (1ำ, $3^{\circ}$ e $5^{\circ}$ trifólios); quatro períodos de tempo entre a aplicação de fungicidas e chuva simulada (0, 30, 60 e 120min.), mais uma testemunha sem chuva; três tratamentos com fungicida: azoxistrobina + ciproconazol

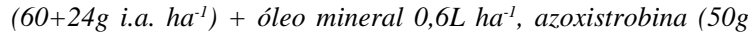
i.a. $\left.h a^{-1}\right)+$ óleo mineral 0,6L ha $a^{-1}$ e ciproconazol (30g i.a. $h a^{-1}$ ), mais uma testemunha sem aplicação. Avaliou-se densidade de gotas por centímetro quadrado, diâmetro mediano volumétrico, diâmetro mediano numérico, amplitude relativa, número de dias para o aparecimento da primeira pústula e a área abaixo da curva de progresso da doença. Verificou-se que gotas de menor DMV proporcionam maior velocidade de absorção de fungicidas. Trifólios mais novos absorvem os fungicidas mais rapidamente. A utilização da mistura de azoxistrobina + ciproconazol proporcionou menor evolução da doença com consequente menor área abaixo da curva de progresso da doença.

Palavras-chave: Phakopsora pachyrhizi, tecnologia de aplicação, estrobilurina, triazol, chuva simulada, residual.

\section{ABSTRACT}

This research aimed to evaluate the effect of large, medium and fine droplets spectra and its interaction with the fungicide absorption rate and leaflets age through indirect measurement expressed by the residual of Asian soybean rust (Phakopsora pachyrhizi) control. The experimental design was composed by completely randomized design with four replications in a factorial $(3 \times 3 \times 5 \times 4)$, which factors were composed of: three drops spectra (large, medium and fine); three fisiological leaflets ages setting on the plant $\left(1^{\text {st }}, 3^{\text {rd }}\right.$ and $5^{\text {th }}$ leaflets), four time periods between the fungicide application and simulated rain (0,30,60 and 120') and a control with no rain; three fungicide treatments with Azoxystrobin+Cyproconazole $\left(60+24 g\right.$ a.i. $\left.h^{-1}\right)+0.6 L h^{-1}$, Azoxystrobin $\left(50 g\right.$ a.i. ha $\left.a^{-1}\right)+$

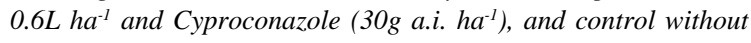
spray. It were evaluated the density of droplets per square centimeter, volume median diameter, number median diameter, relative amplitude, number of days for the first pustule display and the area under disease progress curve. It was found that drops with smaller volume median diameter have higher fungicides absorption rates. Newer leaflets provide more quickly fungicides absorption. The use of Azoxystrobin + cyproconazole provided lower disease progress with consequent lower area under the disease progress curve.

Key words: Phakopsora pachyrhizi, spray technology, strobilurin, triazole, simulated rain, residual.

\section{INTRODUÇÃO}

A ferrugem asiática, causada pelo fungo Phakopsora pachyrhizi Sidow, constitui-se na principal doença da cultura da soja, já que as condições climáticas são favoráveis ao patógeno. Na maioria das regiões, sua alta virulência e velocidade de dispersão são fatores que tornam essa doença altamente agressiva (DEBONA et al., 2008). No que tange aos

IDepartamento de Defesa Fitossanitária, Centro de Ciências Rurais (CCR), Universidade Federal de Santa Maria (UFSM), 97105900, Santa Maria, RS, Brasil. E-mail: giuvanlenz@gmail.com. *Autor para correspondência. 
tecidos vegetais, sabe-se que, com o desenvolvimento da parte aérea, ao mesmo tempo, são encontrados trifólios de diferentes idades que em sua estrutura apresentam diferenças morfológicas e fisiológicas peculiares a cada local. Poucos são os trabalhos na literatura que relacionam a idade físiológica dos tecidos com parâmetros relacionados à infecção de Phakopsora pachyrhizi e à absorção de fungicidas.

Além dessas características, outro fator que interfere na absorção de fungicidas é o espectro de gotas utilizado na pulverização. Segundo PAULSRUD \& MONTGOMERY (2005), a meta da aplicação de fungicidas é produzir um tamanho de gotas que possibilite bom equilíbrio da cobertura, penetração e deposição de gotas.

OZEKI \& KUNZ (1998) ressaltam que a eficiência biológica da aplicação depende da qualidade da cobertura e penetração, bem como a redução das perdas por deriva e evaporação proporcionadas pelo diâmetro de gotas. O maior diâmetro de gotas reduz o arraste pela deriva e a evaporação no trajeto da ponta ao alvo, porém promove uma menor cobertura foliar tratada, ou seja, reduzido número de gotas $\mathrm{cm}^{-2} \mathrm{e}$, também, pode apresentar problemas em virtude da sua tendência ao escorrimento. Logo, gotas de menor diâmetro apresentam maior cobertura da superfície tratada, devido ao maior número de gotas. $\mathrm{cm}^{-2}$, porém apresentam um inconveniente que é a disposição à maior evaporação e arraste pela deriva.

O objetivo do presente trabalho foi avaliar o efeito de três espectros de gotas (grossas, médias e finas) na velocidade de absorção de fungicidas para trifólios de diferentes idades através de medida indireta expressa pelo controle de $\boldsymbol{P}$. pachyrhizi na cultura da soja.

\section{MATERIAL E MÉTODOS}

O experimento foi executado primeiramente em Janeiro e repetido em Março de 2010. O delineamento experimental utilizado foi o inteiramente casualizado, com quatro repetições, em arranjo fatorial $(3 \times 3 \times 5 \times 4)$, cujos fatores foram compostos por: três espectros de gotas (grossa, média e fina), avaliações em três idades fisiológicas de trifólios, posicionado em diferentes locais da planta ( $1^{\circ}, 3^{\circ}$ e $5^{\circ}$ trifólios, contados de baixo para cima); quatro períodos de tempo entre a aplicação dos fungicidas e chuva simulada (0,30,60 e 120min), mais uma testemunha sem chuva, três tratamentos com fungicida: azoxistrobina + ciproconazol $\left(60+24 \mathrm{~g}\right.$ i.a. ha $\left.{ }^{-1}\right)$ + óleo mineral Nimbus ${ }^{\circledR} 0,6 \mathrm{~L} \mathrm{ha}^{-1}$, azoxistrobina (50g i.a. ha $\left.^{-1}\right)+$ óleo mineral Nimbus ${ }^{\circledR} 0,6 \mathrm{~L} \mathrm{ha}^{-1}$ e ciproconazol $\left(30 \mathrm{~g}\right.$ i.a. ha $\left.{ }^{-1}\right)$, mais uma testemunha sem aplicação. As doses dos produtos foram definidas conforme recomendações do fabricante.
Os experimentos foram conduzidos em casa de vegetação, sendo a temperatura regulada através de exaustores e a umidade relativa do ar mantida por um sistema de nebulização computadorizado. Para a semeadura, foram utilizadas seis sementes por vaso da cultivar Asgrow 8000 RR e, após a emergência, mantidas duas plantas de soja por vaso, para aplicação dos tratamentos. As aplicações de fungicidas foram realizadas quando as plantas atingiram o estádio V5 (FEHR, 1971) em caráter preventivo. Foi utilizado pulverizador costal dotado de quatro pontas de pulverização, pressurizado com $\mathrm{CO}_{2}$ e regulado para uma taxa de aplicação de $150 \mathrm{~L} \mathrm{ha}^{-1}$.

Os diferentes espectros de gotas foram obtidos utilizando-se pontas de jatos planos das séries XR110015, XR11002 e XR11005 para obtenção de espectros fino, médio e grosso, respectivamente, variando a pressão e velocidade durante as aplicações, porém mantendo-se sempre a mesma taxa de aplicação (150L ha $\left.{ }^{-1}\right)$, conforme recomendação do fabricante das pontas (Teejet). Em cada uma das repetições dos tratamentos, foram dispostas estacas divididas em três níveis, correspondentes ao primeiro, terceiro e quinto trifólios das plantas. Para cada nível, foi colocado um papel hidrossensível fixado horizontalmente em placas de metal com um atilho de borracha.

A partir dos cartões coletados, foram obtidas imagens digitais através de scanner com resolução de varredura de $1200 \mathrm{dpi}$. As imagens foram submetidas à análise computacional pelo software $\mathrm{CIR}^{\circledR}$, versão 1.5 , para determinação da densidade de gotas (DG), diâmetro mediano volumétrico (DMV), diâmetro mediano numérico (DMN) e amplitude relativa (AR) nos três níveis descritos anteriormente. Os dados destas variáveis, juntamente com o número de dias para o aparecimento da primeira pústula (NDAPP), que, neste artigo, foram somente utilizadas para a construção da matriz de correlação simples entre variáveis. A análise completa desses dados pode ser consultada em LENZ et al. (2011).

Após a aplicação dos tratamentos, conforme programado para cada um, foi simulada chuva, utilizando um simulador desenvolvido e validado por DEBORTOLI (2008), trabalhando com intensidade de $255 \mathrm{~mm} \mathrm{~h}^{-1}$ durante quatro minutos. Logo após a simulação da chuva, os vasos foram conduzidos até a casa de vegetação, onde foram mantidos até o final dos experimentos.

Doze horas após a simulação da chuva, os tratamentos foram submetidos à inoculação de $\boldsymbol{P}$. pachyrhizi com uma solução contendo água, espalhante adesivo (Tween 80-100ppm) e uredósporos de $\boldsymbol{P}$. pachyrhizi na concentração de $2 \times 10^{5}$ esporos $\mathrm{ml}^{-1}$. 
Decorridas 12 horas da inoculação artificial, ao amanhecer, foram iniciadas nebulizações, programadas através de um controlador eletrônico para execução de turno de nebulização de $10 \mathrm{~min}$ hora $^{-1}$, mantendo-se assim condições favoráveis ao desenvolvimento do patógeno. Decorridos dois dias da inoculação das plantas, foram feitas avaliações diárias, com auxílio de lupa, para visualização do aparecimento da primeira pústula esporulada. Nas avaliações, foram consideradas as posições estabelecidas no estudo (primeiro, terceiro e quinto trifólios de cada planta).

A severidade da ferrugem da soja foi determinada através de análise visual aos 14, 21 e 28 dias após a aplicação dos tratamentos, atribuindo um percentual de área foliar com sintomas da doença, utilizando-se como base a escala diagramática para avaliação da ferrugem da soja proposta por GODOY et al. (2006). Com base na severidade observada, procedeu-se ao cálculo da Área Abaixo da Curva de Progresso da Doença (AACPD), segundo CAMPBELL \&MADDEN (1990).

Os dados obtidos a partir dos dois experimentos conduzidos foram submetidos inicialmente à análise conjunta, utilizando-se para tanto o pacote estatístico SOC $^{\circledR}$ versão 2.1. Como a análise conjunta não mostrou diferenças de resposta dos tratamentos nas duas épocas avaliadas, foi efetuada análise de variância a partir da média das duas épocas.

Os resultados obtidos foram submetidos à análise de variância e à interação entre os fatores, bem como suas médias, foram comparadas através do teste de Tukey a 5\% de probabilidade de erro, com a utilização do pacote estatístico Assistat ${ }^{\circledR}$ versão 7.5 beta (SILVA \&AZEVEDO, 2002).

\section{RESULTADOS E DISCUSSÃO}

Área abaixo da curva de progresso da doença (AACPD)

A análise de variância dos dados revelou interação de quarto nível entre os fatores considerados e a área abaixo da curva de progresso da doença ao nível de $1 \%$ de significância. Foi analisada a interação com relação à idade fisiológica dos trifólios, intervalos de simulação de chuva após a aplicação e fungicidas para cada espectro de gotas utilizado.

A tabela 1 mostra o efeito da interação entre a idade fisiológica dos trifólios, intervalos de simulação de chuva após a aplicação de fungicidas para o espectro de gotas grossas na área abaixo da curva de progresso da doença. Inicialmente, considerando as médias para a idade dos trifólios, verificou-se maior severidade da doença nos tecidos mais velhos, evidenciando a menor eficiência de controle causado pela baixa absorção dos produtos. Menor AACPD foi observada sempre que foi aplicada a mistura Azoxistrobina + Ciproconazol, porém, para a maioria das situações, não houve diferença entre os três produtos aplicados. Considerando que as avaliações de severidade da doença foram realizadas aos 14, 21 e 28 dias após a aplicação dos tratamentos, talvez, com o decorrer do ciclo, as diferenças entre os produtos poderiam ser ampliadas. Essa hipótese é considerada levando em conta o fato de que o melhor tratamento perdeu o residual aos 25 dias após a aplicação. Dessa forma, este e muitos outros tratamentos, na última avaliação (28 dias), estariam apenas no início do desenvolvimento da doença.

Essa hipótese é suportada de forma consistente se for considerada a análise de correlação simples entre as variáveis trabalhadas (Tabela 2), que mostra que existe uma alta correlação entre número de dias para o aparecimento da primeira pústula (NDAPP) e a área AACPD, com um coeficiente de -0,8498, significativo ao nível de $1 \%$ de probabilidade. Levandose em conta esse alto grau de correlação, pode-se assumir que, com a evolução da doença, as tendências seriam mantidas, porém, com maior amplitude.

Para o fator chuva e seus níveis, os maiores valores de AACPD foram para os tratamentos que receberam chuva imediatamente após a aplicação, sendo que a interferência verificada foi menor, à medida que se aumentou o intervalo entre a aplicação e a simulação de chuva para todos os trifólios avaliados.

A tabela 1 ainda permite a análise dos fungicidas isolados ou em mistura e o intervalo de chuva. Notou-se que os valores de AACPD para a mistura se mantiveram similares, à medida que o intervalo de chuva foi distanciado da aplicação realizada para cada trifólio. Comportamento diferente foi observado nos tratamentos com os ativos isolados, nos quais a maioria dos valores se aproximou somente nas leituras após os 60 minutos. Essas observações reforçam a importância e o efeito sinérgico da mistura dos dois ativos, aumentando o controle pela maior velocidade de absorção.

Na tabela 3, são evidenciados os efeitos da interação entre a idade fisiológica dos trifólios, intervalos de simulação de chuva após a aplicação e fungicidas para o espectro de gotas médias na área AACPD. Pela análise dos dados das médias da idade dos trifólios, verificou-se mais uma vez que quanto menor a idade dos trifólios, menor a AACPD.

Considerando o desdobramento das interações entre os fatores fungicidas e chuvas dentro de cada idade dos trifólios, para a maioria dos casos, 
Tabela 1 - Área abaixo da curva de progresso de Phakopsora pachyrhizi na interação entre idade fisiológica dos trifólios, intervalos de simulação de chuva após a aplicação e fungicidas para o espectro de gotas grossas.

\begin{tabular}{|c|c|c|c|c|c|}
\hline \multicolumn{6}{|c|}{ Gotas grossas } \\
\hline---- & ---------" & \multirow{2}{*}{ Azox. + cipr. } & 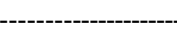 & 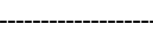 & \\
\hline \multirow[b]{2}{*}{ Test** } & Test.* & & Azox. & Cipr. & \multirow[t]{2}{*}{ Médias } \\
\hline & $101,13 \quad \mathrm{aA}^{* * *}$ & $2,87 \quad b B$ & $5,7 \mathrm{cB}$ & $7,6 \quad \mathrm{bB}$ & \\
\hline $0^{\prime}$ & 102,46 aA & $17,01 \quad \mathrm{aC}$ & $20,91 \mathrm{aC}$ & $33,08 \quad \mathrm{aB}$ & \\
\hline $30^{\prime}$ & 108,50 aA & $6,77 \mathrm{abC}$ & 17,06 abB & $23,94 \quad \mathrm{aB}$ & $34,82 \mathrm{a}$ \\
\hline $60 "$ & 110,08 aA & $4,62 \quad b B$ & 6,77 bcB & $9,94 \quad b B$ & \\
\hline \multirow[t]{2}{*}{$120^{\prime \prime}$} & 111,21 aA & $4,85 \quad b B$ & $6,20 \mathrm{cB}$ & 7,58 bB & \\
\hline & Test. & Azox. + cipr. & Azox. & Cipr. & \\
\hline Test & 54,23 aA & $2,24 \quad b B$ & $2,87 \quad \mathrm{aB}$ & $2,87 \quad b B$ & \\
\hline $0^{\prime}$ & 52,35 aA & $13,30 \quad \mathrm{aC}$ & $10,96 \mathrm{aC}$ & $23,45 \quad \mathrm{aB}$ & \\
\hline $30^{\prime}$ & 56,41 aA & $3,27 \quad b B$ & $7,95 \quad \mathrm{aB}$ & 8,16 bB & $19,20 \mathrm{~b}$ \\
\hline $60 "$ & 58,95 aA & $2,56 \quad b B$ & $4,85 \mathrm{aB}$ & 6,14 bB & \\
\hline \multirow[t]{2}{*}{$120 "$} & 56,96 aA & 3,19 bB & $3,50 \quad \mathrm{aB}$ & 3,76 bB & \\
\hline & Test. & Azox. + cipr. & Azox. & Cipr. & \\
\hline Test & 25,93 aA & $1,61 \quad b B$ & 1,61 bB & $1,93 \mathrm{cB}$ & \\
\hline $0^{\prime}$ & 25,20 aA & $11,06 \quad \mathrm{aB}$ & $6,34 \mathrm{aC}$ & $13,46 \quad \mathrm{aB}$ & \\
\hline $30^{\prime}$ & 29,73 aA & 1,93 & 3,50 abBC & 5,02 & $10,61 \mathrm{c}$ \\
\hline $60 "$ & 24,48 aA & 1,61 & $2,56 \quad b B$ & 3,82 bcB & \\
\hline $120 "$ & 30,71 aA & $1,61 \quad b B$ & $1,61 \quad b B$ & $1,61 \mathrm{cB}$ & \\
\hline Médias & 63,96 A & 5,23 & $6,83 \mathrm{C}$ & $10,16 \quad \mathrm{~B}$ & \\
\hline
\end{tabular}

*Test.: Testemunha para fungicidas; Azox. + cipr.: Azoxistrobina + Ciproconazol; Azox.: Azoxistrobina; Cipr.: Ciproconazol. **Test.: Testemunha para chuva simulada; 0, 30, 60, 120: tempos, em minutos, decorridos entre a aplicação dos tratamentos e a simulação de chuva. ***Médias seguidas pela mesma letra maiúscula nas linhas e minúscula nas colunas não diferem estatisticamente entre si pelo teste de Tukey em nível de $5 \%$ de probabilidade de erro $(0,01=<\mathrm{P}<0,05)$. $* * * *$ A idade está denominada de primeiro a quinto trifólio, sendo que o primeiro trifólio refere-se ao trifólio mais velho.

não foi possível de serem evidenciadas diferenças entre os tratamentos. Porém, a análise das médias mostrou que a maior eficiência de controle foi obtida com a aplicação da mistura de Azoxistrobina + Ciproconazol, seguida de Azoxistrobina e Ciproconazol, aplicados individualmente.
Os níveis de AACPD demonstrados na tabela 4, que considera o espectro de gotas finas, revelam que todos os valores para estes tratamentos foram inferiores, se considerados os demais espectros de gotas utilizados. Da mesma forma que na tabela 3, o desdobramento das interações não permitiu

Tabela 2 - Matriz de correlação simples entre as variáveis analisadas.

\begin{tabular}{|c|c|c|c|c|c|c|}
\hline Variável*** & $\mathrm{NGC}$ & DMV & $\mathrm{DMN}$ & ARE & NDAPP & $A A C P D$ \\
\hline $\mathrm{NGC}$ & 1 & $-0,9666 * *$ & $-0,7252 * *$ & $0,0484^{\text {ns }}$ & $0,4853 * *$ & $-0,5416^{* *}$ \\
\hline DMV & & 1 & $0,7058 * *$ & $-0,078^{\text {ns }}$ & $-0,5054 * *$ & $0,5315^{* *}$ \\
\hline $\mathrm{DMN}$ & & & 1 & $-0,1202^{\text {ns }}$ & $-0,2442^{*}$ & $0,3101 * *$ \\
\hline ARE & & & & 1 & $0,0762^{\mathrm{ns}}$ & $-0,1366^{\mathrm{ns}}$ \\
\hline NDAPP & & & & & 1 & $-0,8498 * *$ \\
\hline AACPD & & & & & & 1 \\
\hline
\end{tabular}

*Significativo ao nível de $5 \%$ de probabilidade $(0,01=<\mathrm{P}<0,05)$; ** Significativo em nível de $1 \%$ de probabilidade $(\mathrm{P}<0,01)$; ${ }^{\text {ns }}$ Não significativo; ***NGC: número de gotas por $\mathrm{cm}^{2}$; DMV: diâmetro mediano volumétrico; DMN: diâmetro mediano numérico; ARE: amplitude relativa; NDAPP: número de dias para o aparecimento da primeira pústula; AACPD: área abaixo da curva de progresso da doença. 
Tabela 3 - Área abaixo da curva de progresso de Phakopsora pachyrhizi na interação entre idade fisiológica dos trifólios, intervalos de simulação de chuva após a aplicação e fungicidas para o espectro de gotas médias.

\begin{tabular}{|c|c|c|c|c|c|}
\hline \multicolumn{6}{|c|}{ Gotas médias } \\
\hline--- & 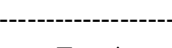 & 1ำ trifól & ..... & 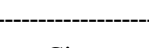 & \\
\hline & Test.* & Azox. + cipr. & Azox. & Cipr. & Médias \\
\hline Test** & $106,31 \mathrm{aA} * * *$ & $2,49 \mathrm{aB}$ & $3,41 \mathrm{bB}$ & $3,41 \mathrm{cB}$ & \\
\hline $0^{\prime}$ & $99,31 \mathrm{aA}$ & $6,04 \mathrm{aC}$ & 7,26abC & $20,78 \mathrm{aB}$ & \\
\hline $30^{\prime}$ & $108,94 \mathrm{aA}$ & $4,16 \mathrm{aC}$ & $12,03 \mathrm{aB}$ & $17,46 \mathrm{abB}$ & $31,94 a$ \\
\hline $60^{\prime \prime}$ & $102,38 \mathrm{aA}$ & $4,29 \mathrm{aC}$ & 7,48abC & $14,18 \mathrm{bB}$ & \\
\hline \multirow[t]{2}{*}{$120^{\prime \prime}$} & $106,09 \mathrm{aA}$ & $1,88 \mathrm{aB}$ & $3,68 \mathrm{bB}$ & $7,22 \mathrm{cB}$ & \\
\hline & Test. & Azox. + cipr. & Azox. & Cipr. & \\
\hline Test & $54,72 \mathrm{aA}$ & $1,58 \mathrm{aB}$ & $2,19 \mathrm{aB}$ & $2,19 \mathrm{bB}$ & \\
\hline $0^{\prime}$ & $51,98 \mathrm{aA}$ & $3,98 \mathrm{aB}$ & $4,38 \mathrm{aB}$ & $80,05 \mathrm{aB}$ & \\
\hline $30^{\prime}$ & $55,13 \mathrm{aA}$ & $2,19 \mathrm{aB}$ & $4,90 \mathrm{aB}$ & $6,87 \mathrm{abB}$ & $16,22 b$ \\
\hline 60" & $51,63 \mathrm{aA}$ & $2,80 \mathrm{aB}$ & $4,29 \mathrm{aB}$ & $5,95 \mathrm{abB}$ & \\
\hline \multirow[t]{2}{*}{$120^{\prime \prime}$} & $48,13 \mathrm{aA}$ & $1,58 \mathrm{aB}$ & $2,19 \mathrm{aB}$ & $4,73 \mathrm{abB}$ & \\
\hline & Test. & Azox. + cipr. & Azox. & Cipr. & \\
\hline Test & $30,54 \mathrm{aA}$ & $1,58 \mathrm{aB}$ & $1,58 \mathrm{aB}$ & $1,58 \mathrm{aB}$ & \\
\hline $0^{\prime}$ & $29,05 \mathrm{aA}$ & $2,80 \mathrm{aB}$ & $2,49 \mathrm{aB}$ & $4,38 \mathrm{aB}$ & \\
\hline $30^{\prime}$ & $31,24 \mathrm{aA}$ & $1,58 \mathrm{aB}$ & $2,19 \mathrm{aB}$ & $2,98 \mathrm{aB}$ & $9,78 \mathrm{c}$ \\
\hline $60^{\prime \prime}$ & $33,95 \mathrm{aA}$ & $1,58 \mathrm{aB}$ & $2,80 \mathrm{aB}$ & $3,59 \mathrm{aB}$ & \\
\hline $120^{\prime \prime}$ & $28,00 \mathrm{aA}$ & $1,58 \mathrm{aB}$ & $1,58 \mathrm{aB}$ & $2,49 \mathrm{aB}$ & \\
\hline Médias & $63,36 \mathrm{~A}$ & $2,67 \mathrm{D}$ & $4,16 \mathrm{C}$ & $7,06 \mathrm{~B}$ & \\
\hline
\end{tabular}

*Test.: Testemunha para fungicidas; Azox. + cipr.: Azoxistrobina + Ciproconazol; Azox.: Azoxistrobina; Cipr.: Ciproconazol. **Test.: Testemunha para chuva simulada; 0,30, 60, 120: tempos, em minutos, decorridos entre a aplicação dos tratamentos e a simulação de chuva. ***Médias seguidas pela mesma letra maiúscula nas linhas e minúscula nas colunas não diferem estatisticamente entre si pelo teste de Tukey em nível de $5 \%$ de probabilidade de erro $(0,01=<\mathrm{P}<0,05)$. $* * * *$ A idade está denominada de primeiro a quinto trifólio, sendo que o primeiro trifólio refere-se ao trifólio mais velho.

diferenciação consistente dos dados, sendo que, dessa forma, serão discutidos os valores médios dos fatores.

Para o fator fungicidas, pode-se perceber que Azoxistrobina + Ciproconazol apresentou a menor AACPD, seguida da aplicação de Azoxistrobina e Ciproconazol, individualmente, que não apresentaram diferença entre si. Semelhante à análise da tabela 3 , as medias da $A A C P D$ foram as menores, à medida que se aumentou o intervalo de tempo entre aplicação e a chuva.

Considerando os níveis do fator espectro de gotas, verificou-se, sem exceção, que o espectro de gotas finas proporcionou melhor eficácia de controle da doença, expresso pela AACPD. Os maiores valores de doença acumulada foram verificados para os trifólios mais velhos, diminuindo conforme foram considerados trifólios mais novos.

A recomendação de espectros de gotas de baixo DMV necessita de especial atenção. Segundo BOLLER (2004), de acordo com o diâmetro das gotas, com a temperatura e a umidade relativa do ar, pode haver maior ou menor perda de defensivos através da evaporação. Quanto menor o diâmetro das gotas, maior a superfície de exposição com o meio e mais acentuada sua evaporação.

Nesse sentido, há que se considerar também que as gotas com diâmetro inferior a $150 \mu \mathrm{m}$ (espectro fino) são facilmente perdidas pelo efeito da deriva, podendo contaminar áreas indesejadas e causar sensíveis prejuízos econômicos e ambientais.

\section{Correlação simples entre variáveis}

A tabela 2 apresenta uma matriz de correlação simples entre as variáveis analisadas. Esse tipo de análise dá uma ideia da correlação carregada entre uma série de variáveis e é um sinal importante da relação de linearidade, seguida pelos resultados dos tratamentos. Em primeira análise, verificam-se elevadas estimativas de correlações, tanto positivas quanto negativas para algumas variáveis estudadas.

A correlação entre o número de gotas por $\mathrm{cm}^{2}$ (NGC) e o diâmetro mediano volumétrico foi a que 
Tabela 4 - Área abaixo da curva de progresso de Phakopsora pachyrhizi na interação entre idade fisiológica dos trifólios, intervalos de simulação de chuva após a aplicação e fungicidas para o espectro de gotas finas.

\begin{tabular}{|c|c|c|c|c|c|}
\hline \multicolumn{6}{|c|}{ Gotas finas } \\
\hline & Test.* & Azox. + cipr. & Azox. & Cipr. & Médias \\
\hline Test** & $108,06 \mathrm{aA}^{* * *}$ & $2,19 \mathrm{aB}$ & $2,49 \mathrm{aB}$ & $2,49 \mathrm{bB}$ & \\
\hline $0^{\prime}$ & $111,56 \mathrm{abA}$ & $2,49 \mathrm{aC}$ & $4,46 \mathrm{aBC}$ & $6,39 \mathrm{aB}$ & \\
\hline $30^{\prime}$ & $112,00 \mathrm{aA}$ & $2,49 \mathrm{aB}$ & $3,72 \mathrm{aB}$ & $4,86 \mathrm{abB}$ & $30,05 a$ \\
\hline $60 "$ & $113,44 \mathrm{aA}$ & $2,49 \mathrm{aB}$ & $3,41 \mathrm{aB}$ & $5,08 \mathrm{abB}$ & \\
\hline \multirow[t]{2}{*}{$120^{\prime \prime}$} & $103,56 \mathrm{aA}$ & $2,80 \mathrm{aB}$ & $2,80 \mathrm{aB}$ & 4,29abB & \\
\hline & Test. & Azox. + cipr. & Azox. & Cipr. & \\
\hline Test & $54,16 \mathrm{aA}$ & $1,58 \mathrm{aB}$ & $1,58 \mathrm{aB}$ & $1,58 \mathrm{aB}$ & \\
\hline $0^{\prime}$ & $51,98 \mathrm{aA}$ & $1,58 \mathrm{aB}$ & $2,80 \mathrm{aB}$ & $3,89 \mathrm{aB}$ & \\
\hline $30^{\prime}$ & $51,98 \mathrm{aA}$ & $1,58 \mathrm{aB}$ & $2,80 \mathrm{aB}$ & $3,28 \mathrm{aB}$ & $14,73 b$ \\
\hline $60^{\prime \prime}$ & $53,33 \mathrm{aA}$ & $1,58 \mathrm{aB}$ & $2,19 \mathrm{aB}$ & $3,28 \mathrm{aB}$ & \\
\hline \multirow[t]{2}{*}{$120^{\prime \prime}$} & $49,09 \mathrm{aA}$ & $1,58 \mathrm{aB}$ & $1,88 \mathrm{aB}$ & $2,89 \mathrm{aB}$ & \\
\hline & Test. & Azox. + cipr. & Azox. & Cipr. & \\
\hline Test & $32,24 \mathrm{aA}$ & $1,31 \mathrm{aB}$ & $1,31 \mathrm{aB}$ & $1,40 \mathrm{aB}$ & \\
\hline $0^{\prime}$ & $28,00 \mathrm{aA}$ & $1,58 \mathrm{aB}$ & $1,58 \mathrm{aB}$ & $2,49 \mathrm{aB}$ & \\
\hline $30^{\prime}$ & $30,06 \mathrm{abA}$ & $1,58 \mathrm{aB}$ & $1,58 \mathrm{aB}$ & $2,19 \mathrm{aB}$ & $8,90 \mathrm{c}$ \\
\hline $60^{\prime \prime}$ & $32,94 \mathrm{aA}$ & $1,40 \mathrm{aB}$ & $1,58 \mathrm{aB}$ & $2,19 \mathrm{aB}$ & \\
\hline $120^{\prime \prime}$ & $29,88 \mathrm{abA}$ & $1,40 \mathrm{aB}$ & $1,49 \mathrm{aB}$ & $1,88 \mathrm{aB}$ & \\
\hline Médias & $64,15 \mathrm{~A}$ & $1,84 \mathrm{C}$ & $2,38 \mathrm{BC}$ & $3,21 \mathrm{~B}$ & \\
\hline
\end{tabular}

*Test.: Testemunha para fungicidas; Azox. + cipr.: Azoxistrobina + Ciproconazol; Azox.: Azoxistrobina; Cipr.: Ciproconazol. **Test.: Testemunha para chuva simulada; 0, 30, 60, 120: tempos, em minutos, decorridos entre a aplicação dos tratamentos e a simulação de chuva. ***Médias seguidas pela mesma letra maiúscula nas linhas e minúscula nas colunas não diferem estatisticamente entre si pelo teste de Tukey em nível de $5 \%$ de probabilidade de erro $(0,01=<\mathrm{P}<0,05)$. $* * * *$ A idade está denominada de primeiro a quinto trifólio, sendo que o primeiro trifólio refere-se ao trifólio mais velho.

apresentou maior coeficiente de linearidade. Essa correlação já é verificada de longa data por diversos trabalhos, envolvendo tecnologia de aplicação de produtos líquidos (BOLLER, 2007; COSTA, 2009) que afirmam que, quanto menor o diâmetro mediano volumétrico das gotas geradas, maior a área de cobertura das superfícies, expressa pelo número de gotas por $\mathrm{cm}^{2}$.

O segundo maior coeficiente de linearidade da correlação foi verificado na interação entre o número de dias para o aparecimento da primeira pústula (NDAPP) e a AACPD. Como esse coeficiente demonstra que a resposta dos valores de doença acumulada da AACPD são explicados por aproximadamente $85 \%$ do valor do número de dias para o aparecimento da primeira pústula, foi levantada a hipótese, na discussão da AACPD, de que, em função das avaliações de severidade terem prosseguido somente até 28 dias após a aplicação, devido ao fato de que as testemunhas já apresentavam os primeiros trifólios em processo de senescência quase total, os valores de severidade poderiam manter a mesma tendência de crescimento, para os demais tratamentos, porém com maior amplitude de distanciamento, permitindo, assim, a diferenciação entre eles.

Com exceção da amplitude relativa, que não apresentou correlação significativa com nenhuma das demais variáveis, todos os demais coeficientes calculados tiveram variação de resposta compreendida entre 50 e $70 \%$, aproximadamente, com nível de significância de 1\%, com exceção da correlação entre diâmetro mediano numérico (DMN) e NADPP, que apresentou coeficiente de aproximadamente $25 \%$ e com $5 \%$ de significância.

\section{CONCLUSÃO}

Gotas de menor diâmetro mediano volumétrico apresentaram maior velocidade de absorção de fungicidas. Trifólios mais novos absorvem os fungicidas mais rapidamente e estabelecem um maior atraso no desenvolvimento da doença. A utilização da 
mistura de azoxistrobina + ciproconazol proporciona menor evolução e acúmulo final de doença. A influência da chuva sobre o período de proteção das plantas possui relação altamente dependente do intervalo entre a aplicação dos produtos e a ocorrência dela, além do produto que está sendo aplicado e da idade dos tecidos considerados.

\section{REFERÊNCIAS}

BOLLER, W. Parâmetros técnicos para seleção de pontas. In: BORGES, L.D. Tecnologia de aplicação de defensivos agrícolas. Passo Fundo: Plantio Direto Eventos, 2004. p.37-52.

BOLLER, W. Resposta da tecnologia de aplicação de defensivos em relação à concepção atmosférica visando o controle de doenças de plantas. Summa Phytopathologica, v.33, supl., p.113-116, 2007.

CAMPBELL, C.L.; MADDEN, L.V. Introduction to plant disease epidemiology. New York: Wiley, 1990. 532p.

COSTA, D.I. Eficiência e qualidade das aplicações de fungicidas, por vias terrestre e aérea, no controle de doenças foliares e no rendimento de grãos de soja e milho. 2009. 144f. Tese (Doutorado em Agronomia) Universidade de Passo Fundo, Passo Fundo, RS.

DEBONA, D. et al. Efeito de níveis de cálcio e sombreamento em plantas de soja sobre a infecção por Phakopsora pachyrhizi. Tropical Plant Pathology, v.33, n.5, p.388-389, 2008. Disponível em: <http://www.scielo.br/scielo.php?pid=S1982$56762008000500009 \&$ script $=$ sci_arttext $>$. Acesso em: 03 set. 2010. doi: 10.1590/S1982-56762008000500009.
DEBORTOLI, M.P. Efeito do “rainfastness" e adjuvante na aplicação de fungicidas foliares em cultivares de soja. 2008. 57f. Dissertação (Mestrado em Engenharia Agrícola) Universidade Federal de Santa Maria, Santa Maria, RS.

FEHR, W.R. et al. Stage of development descriptions for soybeans, Glycine $\max (\mathrm{L}$.) Merrill. Crop Science, v.11, n.6, p.929-931, 1971.

GODOY, C.V. et al. Diagrammatic scale for assessment of soybean rust severity. Fitopatologia Brasileira, v.31, n.1, p.63-68, 2006.

LENZ, G. et al. Espectro de gotas e idade de trifólios na taxa de absorção e efeito residual de fungicidas em soja. Ciência Rural, v.41, n.10, p.1702-1708, 2011. Disponível em: <http:// www.scielo.br/scielo.php? script $=$ sci_arttext\&pid $=$ S0103$84782011001000004 \& \operatorname{lng}=\mathrm{en} \& \mathrm{nrm}=\mathrm{iso}>$. Acesso em: 29 maio, 2012. doi: 10.1590/S0103-84782011005000127.

OZEKI, Y.; KUNZ, R.P. Tecnologia de aplicação aérea aspectos práticos. In: GUEDES, J.V.C.; DORNELLES S.H.B. (Org.). Tecnologia e segurança na aplicação de agrotóxicos: novas tecnologias. Santa Maria: UFSM, 1998. p.65-78.

PAULSRUD, B.E.; MONTGOMERY, M. Characteristics of fungicides used in field crops. Report on Plant Disease. n.1002, p.38, 2005.

SILVA, F.A.S.; AZEVEDO, C.A.V. Versão do programa computacional Assistat para o sistema operacional Windows. Revista Brasileira de Produtos Agroindustriais, v.4, n.1, p.71-78, 2002 . 